\title{
Strongly modular lattices with long shadow
}

\author{
par GABRIELE NEBE
}

\begin{abstract}
RÉSUmÉ. Cet article donne une classification des réseaux fortement modulaires dont la longueur de l'ombre prend les deux plus grandes valeurs possibles.
\end{abstract}

ABstract. This article classifies the strongly modular lattices with longest and second longest possible shadow.

\section{Introduction}

To an integral lattice $L$ in the euclidean space $\left(\mathbb{R}^{n},(),\right)$, one associates the set of characteristic vectors $v \in \mathbb{R}^{n}$ with $(v, x) \equiv(x, x) \bmod 2 \mathbb{Z}$ for all $x \in L$. They form a coset modulo $2 L^{*}$, where

$$
L^{*}=\left\{v \in \mathbb{R}^{n} \mid(v, x) \in \mathbb{Z} \forall x \in L\right\}
$$

is the dual lattice of $L$. Recall that $L$ is called integral, if $L \subset L^{*}$ and unimodular, if $L=L^{*}$. For a unimodular lattice, the square length of a characteristic vector is congruent to $n$ modulo 8 and there is always a characteristic vector of square length $\leq n$. In [1] Elkies characterized the standard lattice $\mathbb{Z}^{n}$ as the unique unimodular lattice of dimension $n$, for which all characteristic vectors have square length $\geq n$. [2] gives the short list of unimodular lattices $L$ with $\min (L) \geq 2$ such that all characteristic vectors of $L$ have length $\geq n-8$. The largest dimension $n$ is 23 and in dimension 23 this lattice is the shorter Leech lattice $O_{23}$ of minimum 3. In this paper, these theorems are generalized to certain strongly modular lattices. Following [7] and [8], an integral lattice $L$ is called $N$-modular, if $L$ is isometric to its rescaled dual lattice $\sqrt{N} L^{*}$. A $N$-modular lattice $L$ is called strongly $N$-modular, if $L$ is isometric to all rescaled partial dual lattices $\sqrt{m} L^{*, m}$, for all exact divisors $m$ of $N$, where

$$
L^{*, m}:=L^{*} \cap \frac{1}{m} L .
$$

The simplest strongly $N$-modular lattice is

$$
C_{N}:=\perp_{d \mid N} \sqrt{d} \mathbb{Z}
$$

Manuscrit reçu le 10 septembre 2002. 
of dimension $\sigma_{0}(N):=\sum_{d \mid N} 1$ the number of divisors of $N$. The lattice $C_{N}$ plays the role of $\mathbb{Z}=C_{1}$ for square free $N>1$.

With the help of modular forms Quebbemann [8] shows that for

$$
N \in \mathcal{L}:=\{1,2,3,5,6,7,11,14,15,23\}
$$

(which is the set of all positive integers $N$ such that the sum of divisors

$$
\sigma_{1}(N):=\sum_{d \mid N} d
$$

divides 24), the minimum of an even strongly $N$-modular lattice $L$ of dimension $n$ satisfies

$$
\min (L) \leq 2+2\left\lfloor\frac{n \sigma_{1}(N)}{24 \sigma_{0}(N)}\right\rfloor .
$$

Strongly modular lattices meeting this bound are called extremal. Whereas Quebbemann restricts to even lattices, [9] shows that the same bound also holds for odd strongly modular lattices, where there is one exceptional dimension $n=\sigma_{0}(N)\left(\frac{24}{\sigma_{1}(N)}-1\right)$, where the bound on the minimum is 3 (and not 2). In this dimension, there is a unique lattice $S^{(N)}$ of minimum 3. For $N=1$, this is again the shorter Leech lattice $O_{23}$. The main tool to get the bound for odd lattices is the shadow

$$
S(L):=\left\{\frac{v}{2} \mid v \text { is a characteristic vector of } L\right\} .
$$

If $L$ is even, then $S(L)=L^{*}$ and if $L$ is odd, $S(L)=L_{0}^{*}-L^{*}$, where

$$
L_{0}:=\{v \in L \mid(v, v) \in 2 \mathbb{Z}\}
$$

is the even sublattice of $L$.

For $N \in \mathcal{L}$ let

$$
s(N):=\frac{24}{\sigma_{1}(N)} .
$$

The main result of this paper is Theorem 3. It is shown that for a strongly $N$-modular lattice $L$ that is rationally equivalent to $C_{N}^{k}$, the minimum

$$
\min _{0}(S(L)):=\min \{(v, v) \mid v \in S(L)\}
$$

equals

$$
M^{(N)}(m, k):= \begin{cases}\frac{1}{N}\left(k \frac{\sigma_{1}(N)}{4}-2 m\right) & \text { if } N \text { is odd } \\ \frac{1}{N}\left(k \frac{\sigma_{1}(N / 2)}{2}-m\right) & \text { if } N \text { is even }\end{cases}
$$

for some $m \in \mathbb{Z}_{\geq 0}$. If $\min _{0}(S(L))=M^{(N)}(0, k)$, then $L \cong C_{N}^{k}$. For the next smaller possible minimum $\min _{0}(S(L))=M^{(N)}(1, k)$ one gets that $L \cong C_{N}^{l} \perp L^{\prime}$, where $\min \left(L^{\prime}\right)>1$ and $\operatorname{dim}\left(L^{\prime}\right) \leq \sigma_{0}(N)(s(N)-1)$ for odd $N$ resp. $\operatorname{dim}\left(L^{\prime}\right) \leq \sigma_{0}(N) s(N)$ for even $N$. The lattices $L^{\prime}$ of maximal possible dimensions have minimum 3 and are uniquely determined: $L^{\prime}=S^{(N)}$, if $N$ 
is odd and $L^{\prime}=O^{(N)}$ (the "odd analogue" of the unique extremal strongly $N$-modular lattice of dimension $\sigma_{0}(N) s(N)$ ) if $N$ is even (see [9, Table 1]).

The main tool to prove this theorem are the formulas for the theta series of a strongly $N$-modular lattice $L$ and of its shadow $S(L)$ developed in [9]. Therefore we briefly repeat these formulas in the next section.

\section{Theta series}

For a subset $S \subset \mathbb{R}^{n}$, which is a finite union of cosets of an integral lattice we put its theta series

$$
\Theta_{S}(z):=\sum_{v \in S} q^{(v, v)}, \quad q=\exp (\pi i z) .
$$

The theta series of strongly $N$-modular lattices are modular forms for a certain discrete subgroup $\Gamma_{N}$ of $S L_{2}(\mathbb{R})$ (see [9]). Fix $N \in \mathcal{L}$ and put

$$
g_{1}^{(N)}(z):=\Theta_{C_{N}}(z)=1+2 q+2 \operatorname{ev}(N) q^{2}+\ldots
$$

where

$$
\operatorname{ev}(N):=\left\{\begin{array}{ll}
1 & \text { if } N \text { is even } \\
0 & \text { if } N \text { is odd }
\end{array} .\right.
$$

Let $\eta$ be the Dedekind eta-function

$$
\eta(z):=q^{\frac{1}{12}} \prod_{m=1}^{\infty}\left(1-q^{2 m}\right), \quad q=\exp (\pi i z) .
$$

and put

$$
\eta^{(N)}(z):=\prod_{d \mid N} \eta(d z)
$$

If $N$ is odd define

$$
g_{2}^{(N)}(z):=\left(\frac{\eta^{(N)}(z / 2) \eta^{(N)}(2 z)}{\eta^{(N)}(z)^{2}}\right)^{s(N)}
$$

and if $N$ is even then

$$
g_{2}^{(N)}(z):=\left(\frac{\eta^{(N / 2)}(z / 2) \eta^{(N / 2)}(4 z)}{\eta^{(N / 2)}(z) \eta^{(N / 2)}(2 z)}\right)^{s(N)} .
$$

Then $g_{2}^{(N)}$ generates the field of modular functions of $\Gamma_{N}$. It is a power series in $q$ starting with

$$
g_{2}^{(N)}(z)=q-s(N) q^{2}+\ldots
$$


Theorem 1. ([9, Theorem 9, Corollary 3]) Let $N \in \mathcal{L}$ and $L$ be a strongly $N$-modular lattice that is rational equivalent to $C_{N}^{k}$. Define $l_{N}:=\frac{1}{8} \sigma_{1}(N)$, if $N$ is odd and $l_{N}:=\frac{1}{6} \sigma_{1}(N)$, if $N$ is even. Then

$$
\Theta_{L}(z)=g_{1}^{(N)}(z)^{k} \sum_{i=0}^{\left\lfloor k l_{N}\right\rfloor} c_{i} g_{2}^{(N)}(z)^{i}
$$

for $c_{i} \in \mathbb{R}$. The theta series of the rescaled shadow $S:=\sqrt{N} S(L)$ of $L$ is

$$
\Theta_{S}(z)=s_{1}^{(N)}(z)^{k} \sum_{i=0}^{\left\lfloor k l_{N}\right\rfloor} c_{i} s_{2}^{(N)}(z)^{i}
$$

where $s_{1}^{(N)}$ and $s_{2}^{(N)}$ are the corresponding "shadows" of $g_{1}^{(N)}$ and $g_{2}^{(N)}$.

For odd $N$

$$
s_{1}^{(N)}(z)=2^{\sigma_{0}(N)} \frac{\eta^{(N)}(2 z)^{2}}{\eta^{(N)}(z)}
$$

and

$$
s_{2}^{(N)}(z)=-2^{-s(N) \sigma_{0}(N) / 2}\left(\frac{\eta^{(N)}(z)}{\eta^{(N)}(2 z)}\right)^{s(N)}
$$

For $N=2$ one has

$$
s_{1}^{(2)}(z)=\frac{2 \eta(z)^{5} \eta(4 z)^{2}}{\eta(z / 2)^{2} \eta(2 z)^{3}}
$$

and

$$
s_{2}^{(2)}(z)=-\frac{1}{16}\left(\frac{\eta(z / 2) \eta(2 z)^{2}}{\eta(z)^{2} \eta(4 z)}\right)^{8}
$$

which yields $s_{1}^{(N)}$ and $s_{2}^{(N)}$ for $N=6,14$ as

$$
s_{1}^{(N)}=s_{1}^{(2)}(z) s_{1}^{(2)}\left(\frac{N}{2} z\right)
$$

and

$$
s_{2}^{(N)}=\left(s_{2}^{(2)}(z) s_{2}^{(2)}\left(\frac{N}{2} z\right)\right)^{s(N) / s(2)} .
$$

If $N$ is odd, then $s_{1}^{(N)}$ starts with $q^{\sigma_{1}(N) / 4}$ and $s_{2}^{(N)}$ starts with $q^{-2}$. If $N$ is even, then $s_{1}^{(N)}$ starts with $q^{\sigma_{1}\left(\frac{N}{2}\right) / 2}$ and $s_{2}^{(N)}$ starts with $q^{-1}$.

\section{Strongly modular lattices with long shadow.}

Proposition 2. Let $N \in \mathbb{N}$ be square free and let $L$ be a strongly $N$ modular lattice. If $L$ contains a vector of length 1 , then $L$ has an orthogonal summand $C_{N}$. 
Proof. Since $L$ is an integral lattice that contains a vector of length 1 , the unimodular lattice $\mathbb{Z}$ is an orthogonal summand of $L$. Hence $L=\mathbb{Z} \perp L^{\prime}$. If $d$ is a divisor of $N$, then

$$
L \cong \sqrt{d} L^{*, d}=\sqrt{d} \mathbb{Z} \perp \sqrt{d}\left(L^{\prime}\right)^{*, d}
$$

by assumption. Hence $L$ contains an orthogonal summand $\sqrt{d} \mathbb{Z}$ for all divisors $d$ of $N$ and therefore $C_{N}$ is an orthogonal summand of $L$.

Theorem 3. (see [2] for $N=1$ ) Let $N \in \mathcal{L}$ and $L$ be a strongly $N$-modular lattice that is rational equivalent to $C_{N}^{k}$. Let $M^{(N)}(m, k)$ be as defined in the introduction.

(i) $\min _{0}(S(L))=M^{(N)}(m, k)$ for some $m \in \mathbb{Z}_{\geq 0}$.

(ii) If $\min _{0}(S(L))=M^{(N)}(0, k)$ then $L \cong C_{N}^{k}$.

(iii) If $\min _{0}(S(L))=M^{(N)}(m, k)$ then $L \cong C_{N}^{a} \perp L^{\prime}$, where $L^{\prime}$ is a strongly $N$-modular lattice rational equivalent to $C_{N}^{k-a}$ with $\min \left(L^{\prime}\right) \geq 2$ and $\min _{0}\left(S\left(L^{\prime}\right)\right)=M^{(N)}(m, k-a)$.

(iv) If $\min _{0}(S(L))=M^{(N)}(m, 1)$ and $\min (L) \geq 2$, then the number of vectors of length 2 in $L$ is

$$
2 k(s(N)+\operatorname{ev}(N)-(k+1)) .
$$

In particular $k \leq k_{\max }(N)$ with

$$
k_{\max }(N)=s(N)-1+\operatorname{ev}(N)
$$

and if $k=k_{\max }(N)$, then $\min (L) \geq 3$.

Proof. (i) Follows immediately from Theorem 1.

(ii) In this case the theta series of $L$ is $g_{1}^{k}$. In particular $L$ contains $2 k$ vectors of norm 1. Applying Proposition 2 one finds that $L \cong C_{N}$.

(iii) Follows from Proposition 2 and Theorem 1.

(iv) Since $\min (L)>1, \Theta_{L}=g_{1}^{k}-2 k g_{1}^{k} g_{2}$. Explicit calculations give the number of norm-2-vectors in $L$.

The following table gives the maximal dimension $n_{\max }(N)=$ $\sigma_{0}(N) k_{\max }(N)$ of a lattice in Theorem 3 (iv).

\begin{tabular}{|c|c|c|c|c|c|c|c|c|c|c|}
\hline$N$ & 1 & 2 & 3 & 5 & 6 & 7 & 11 & 14 & 15 & 23 \\
\hline$\sigma_{1}(N)$ & 1 & 3 & 4 & 6 & 12 & 8 & 12 & 24 & 24 & 24 \\
\hline$k_{\max }(N)$ & 23 & 8 & 5 & 3 & 2 & 2 & 1 & 1 & 0 & 0 \\
\hline$n_{\max }(N)$ & 23 & 16 & 10 & 6 & 8 & 4 & 2 & 4 & 0 & 0 \\
\hline
\end{tabular}

The lattices $L$ with $\min _{0}(S(L))=M^{(N)}(1, k)$ are listed in an appendix. These are only finitely many since $k$ is bounded by $k_{\max }$. In general it is an open problem whether for all $m$, there are only finitely many strongly $N$-modular lattices $L$ rational equivalent to $C_{N}^{k}$ for some $k$ and of minimum 
$\min (L)>1$ such that $\min _{0}(S(L))=M^{(N)}(m, k)$. For $N=1$, Gaulter [3] proved that $k \leq 2907$ for $m=2$ and $k \leq 8388630$ for $m=3$.

Theorem 4. (cf. [2] for $N=1$ ) Let $N \in \mathcal{L}$ be odd and $k \in \mathbb{N}$ such that

$$
\frac{8}{\sigma_{1}(N)} \leq k \leq k_{\max }(N)=\frac{24}{\sigma_{1}(N)}-1 \text {. }
$$

Then there is a unique strongly $N$-modular lattice $L:=L_{k}(N)$ that is rational equivalent to $C_{N}^{k}$ such that $\min (L)>1$ and $\min _{0}(S(L))=M^{(N)}(1, k)$, except for $N=1$, where there is no such lattice in dimension 9, 10, 11, 13 and there are two lattices in dimension 18 and 20 (see [2]). If $k=k_{\max }(N)$, then $L$ is the shorter lattice $L=S^{(N)}$ described in [9, Table 1] and $\min (L)=3$.

Proof. For $N=15$ and $N=23$ there is nothing to show since $k_{\max }(N)=0$. The case $N=1$ is already shown in [2]. It remains to consider $N \in$ $\{3,5,7,11\}$. Since $N$ is a prime, there are only 2 genera of strongly modular lattices, one consisting of even lattices and one of odd lattices. With a short MAGMA program using Kneser's neighboring method, one obtains a list of all lattices in the relevant genus. In all cases there is a unique lattice with the right number of vectors of length 2 . Gram matrices of these lattices are given in the appendix.

Remark 5. For $N=1$ and dimension $n=9,10,11$ the theta series of the hypothetical shadow has non integral resp. odd coefficients, so there is no lattice $L_{n}(1)$.

Theorem 6. Let $N \in \mathcal{L}$ be even and $k \in \mathbb{N}$ such that

$$
\frac{2}{\sigma_{1}(N / 2)} \leq k \leq k_{\max }(N)=\frac{24}{\sigma_{1}(N)} .
$$

If $(k, N) \neq(3,2)$ then there are strongly $N$-modular lattices $L:=L_{k}(N)$ that are rational equivalent to $C_{N}^{k}$ such that $\min (L)>1$ and $\min _{0}(S(L))=$ $M^{(N)}(1, k)$. If $k=k_{\max }(N)$, then $L_{k}(N)$ is unique. It is the odd lattice $L=O^{(N)}$ described in $[9$, Table 1] and $\min (L)=3$.

Remark 7. For $N=2$ and $k=3$ the corresponding shadow modular form has non integral coefficients, so there is no lattice $L_{3}(2)$.

Remark 8. All odd lattices $L_{k}(N)$ in Theorem 6 lie in the genus of $C_{N}^{k}$.

4. Appendix: The lattices $L_{k}(N)$.

The lattices $L_{k}(1)$ :

The lattices $L_{k}(1)$ are already listed in [2]. They are uniquely determined by their root-sublattices $R_{k}$ and given in the following table:

\begin{tabular}{|c|c|c|c|c|c|c|c|c|c|c|c|c|}
\hline$k$ & 8 & 12 & 14 & 15 & 16 & 17 & 18 & 19 & 20 & 21 & 22 & 23 \\
\hline$R_{k}$ & $E_{8}$ & $D_{12}$ & $E_{7}^{2}$ & $A_{15}$ & $D_{8}^{2}$ & $A_{11} E_{6}$ & $D_{6}^{3}, A_{9}^{2}$ & $A_{7}^{2} D_{5}$ & $D_{4}^{5}, A_{5}^{4}$ & $A_{3}^{7}$ & $A_{1}^{22}$ & 0 \\
\hline
\end{tabular}


The lattices $L_{k}(N)$ for $N>1$ odd:

$L_{2}(3):\left(\begin{array}{cc}2 & 1 \\ 1 & 2\end{array}\right) \perp\left(\begin{array}{cc}2 & 1 \\ 1 & 2\end{array}\right) \cong A_{2} \perp A_{2}$.

Automorphism group: $D_{12} \prec C_{2}$.

$L_{3}(3):\left(\begin{array}{cccccc}2 & 1 & 1 & 1 & 1 & 1 \\ 1 & 2 & 1 & 1 & 1 & 1 \\ 1 & 1 & 2 & 1 & 1 & 1 \\ 1 & 1 & 1 & 3 & 0 & 0 \\ 1 & 1 & 1 & 0 & 3 & 0 \\ 1 & 1 & 1 & 0 & 0 & 3\end{array}\right)$.

Automorphism group: order 1152 .

$L_{4}(3):\left(\begin{array}{cccccccc}2 & 0 & 0 & 0 & -1 & -1 & 0 & 1 \\ 0 & 2 & 0 & 0 & 1 & 1 & 1 & 0 \\ 0 & 0 & 2 & 0 & 0 & -1 & -1 & -1 \\ 0 & 0 & 0 & 2 & -1 & 0 & -1 & -1 \\ -1 & 1 & 0 & -1 & 3 & 1 & 1 & 0 \\ -1 & 1 & -1 & 0 & 1 & 3 & 1 & 0 \\ 0 & 1 & -1 & -1 & 1 & 1 & 3 & 1 \\ 1 & 0 & -1 & -1 & 0 & 0 & 1 & 3\end{array}\right)$.

Automorphism group: order 6144 .

$L_{5}(3):\left(\begin{array}{cccccccccc}3 & -1 & -1 & -1 & 0 & -1 & -1 & -1 & 1 & 0 \\ -1 & 3 & 1 & -1 & -1 & 1 & -1 & 1 & 1 & 0 \\ -1 & 1 & 3 & 1 & 1 & 1 & -1 & 1 & 0 & -1 \\ -1 & -1 & 1 & 3 & 1 & 0 & 1 & 1 & -1 & -1 \\ 0 & -1 & 1 & 1 & 3 & 1 & 0 & -1 & -1 & 0 \\ -1 & 1 & 1 & 0 & 1 & 3 & -1 & 0 & -1 & -1 \\ -1 & -1 & -1 & 1 & 0 & -1 & 3 & 0 & -1 & 1 \\ -1 & 1 & 1 & 1 & -1 & 0 & 0 & 3 & 1 & 0 \\ 1 & 1 & 0 & -1 & -1 & -1 & -1 & 1 & 3 & 1 \\ 0 & 0 & -1 & -1 & 0 & -1 & 1 & 0 & 1 & 3\end{array}\right)$.

Automorphism group: $\pm U_{4}(2) .2$ of order 103680 .

$L_{2}(5):\left(\begin{array}{cc}3 & 1 \\ 1 & 2\end{array}\right) \perp\left(\begin{array}{cc}3 & 1 \\ 1 & 2\end{array}\right)$.

Automorphism group: $\left( \pm C_{2}\right) \prec C_{2}$ of order 32 .

$L_{3}(5):\left(\begin{array}{cccccc}3 & -1 & 1 & -1 & 1 & 0 \\ -1 & 3 & -1 & 0 & 1 & 1 \\ 1 & -1 & 3 & 1 & 0 & 1 \\ -1 & 0 & 1 & 3 & -1 & 1 \\ 1 & 1 & 0 & -1 & 3 & 1 \\ 0 & 1 & 1 & 1 & 1 & 3\end{array}\right)$.

Automorphism group: $\pm S_{5}$ of order 240 . 
$L_{1}(7):\left(\begin{array}{cc}2 & 1 \\ 1 & 4\end{array}\right)$

Automorphism group: $\pm C_{2}$.

$L_{2}(7):\left(\begin{array}{cccc}3 & -1 & 1 & 0 \\ -1 & 3 & 0 & 1 \\ 1 & 0 & 3 & 1 \\ 0 & 1 & 1 & 3\end{array}\right)$.

Automorphism group: order 16 .

$L_{1}(11):\left(\begin{array}{cc}2 & 1 \\ 1 & 6\end{array}\right)$.

Automorphism group: $\pm C_{2}$.

The lattices $L_{k}(N)$ for $N$ even:

For $N=2$ there is only one genus of odd lattices to be considered. Also for $N=14$ there is only one odd genus for each $k$, since 2 is a square modulo 7. For $N=6$, there are 2 such genera, since $L:=(\sqrt{2} \mathbb{Z})^{2} \perp(\sqrt{3} \mathbb{Z})^{2}$ is not in the genus of $C_{6}$. The genus of $L$ contains no strongly modular lattices. The genus of $L \perp C_{6}$ contains 3 lattices with minimum 3, none of which is strongly modular.

$L_{2}(2): L_{2}(2)=D_{4}$ with automorphism group $W\left(F_{4}\right)$ of order 1152 .

$L_{4}(2):\left(\begin{array}{cccccccc}2 & 0 & 0 & 0 & 0 & -1 & -1 & 1 \\ 0 & 2 & 0 & 0 & 0 & 1 & 1 & -1 \\ 0 & 0 & 2 & -1 & 1 & 1 & 1 & -1 \\ 0 & 0 & -1 & 2 & -1 & -1 & 0 & 1 \\ 0 & 0 & 1 & -1 & 2 & 1 & 0 & -1 \\ -1 & 1 & 1 & -1 & 1 & 3 & 2 & -2 \\ -1 & 1 & 1 & 0 & 0 & 2 & 3 & -1 \\ 1 & -1 & -1 & 1 & -1 & -2 & -1 & 3\end{array}\right)$.

The root sublattice is $D_{4} \perp A_{1}^{4}$ and the automorphism group of $L_{4}(2)$ is $W\left(F_{4}\right) \times\left(C_{2}^{4}: D_{8}\right)$ of order 147456 .

$L_{5}(2):\left(\begin{array}{cccccccccc}2 & 1 & 1 & 1 & 1 & 1 & 1 & 1 & 1 & 1 \\ 1 & 2 & 1 & 1 & 1 & 1 & 1 & 1 & 1 & 1 \\ 1 & 1 & 2 & 1 & 1 & 1 & 1 & 1 & 1 & 1 \\ 1 & 1 & 1 & 2 & 1 & 0 & 0 & 0 & 0 & 0 \\ 1 & 1 & 1 & 1 & 2 & 1 & 1 & 1 & 1 & 1 \\ 1 & 1 & 1 & 0 & 1 & 3 & 1 & 1 & 1 & 1 \\ 1 & 1 & 1 & 0 & 1 & 1 & 3 & 1 & 1 & 1 \\ 1 & 1 & 1 & 0 & 1 & 1 & 1 & 3 & 1 & 1 \\ 1 & 1 & 1 & 0 & 1 & 1 & 1 & 1 & 3 & 1 \\ 1 & 1 & 1 & 0 & 1 & 1 & 1 & 1 & 1 & 3\end{array}\right)$

The root sublattice is $A_{5}$ and the automorphism group of $L_{5}(2)$ is $\pm S_{6} \times S_{6}$ of order 1036800 . 
$L_{6}(2):$ There are two such lattices:

$$
L_{6 a}(2):\left(\begin{array}{cccccccccccc}
2 & 0 & 1 & 1 & 0 & 0 & 1 & 1 & 1 & 1 & -1 & -1 \\
0 & 2 & 0 & 0 & -1 & 1 & 1 & 1 & -1 & 1 & 0 & -1 \\
1 & 0 & 2 & 1 & 0 & 0 & 1 & 1 & 1 & 1 & -1 & -1 \\
1 & 0 & 1 & 2 & 0 & 0 & 1 & 1 & 1 & 1 & -1 & -1 \\
0 & -1 & 0 & 0 & 2 & -1 & 0 & -1 & 1 & -1 & 1 & 1 \\
0 & 1 & 0 & 0 & -1 & 2 & 0 & 1 & -1 & 1 & 0 & -1 \\
1 & 1 & 1 & 1 & 0 & 0 & 3 & 1 & 0 & 1 & 0 & -1 \\
1 & 1 & 1 & 1 & -1 & 1 & 1 & 3 & 0 & 1 & -1 & -1 \\
1 & -1 & 1 & 1 & 1 & -1 & 0 & 0 & 3 & 0 & 0 & 0 \\
1 & 1 & 1 & 1 & -1 & 1 & 1 & 1 & 0 & 3 & -1 & -1 \\
-1 & 0 & -1 & -1 & 1 & 0 & 0 & -1 & 0 & -1 & 3 & 1 \\
-1 & -1 & -1 & -1 & 1 & -1 & -1 & -1 & 0 & -1 & 1 & 3
\end{array}\right)
$$

and

$$
L_{6 b}(2):\left(\begin{array}{cccccccccccc}
2 & 0 & 0 & 0 & 0 & 0 & 1 & 1 & -1 & -1 & 1 & 1 \\
0 & 2 & 0 & 0 & 0 & 0 & 0 & 0 & 0 & 0 & 1 & 1 \\
0 & 0 & 2 & 0 & 0 & 0 & 1 & -1 & 0 & 1 & -1 & -1 \\
0 & 0 & 0 & 2 & 0 & 0 & 0 & 0 & 1 & 0 & 0 & 0 \\
0 & 0 & 0 & 0 & 2 & 0 & -1 & 1 & 0 & -1 & 1 & 1 \\
0 & 0 & 0 & 0 & 0 & 2 & 0 & 0 & -1 & 0 & 1 & 1 \\
1 & 0 & 1 & 0 & -1 & 0 & 3 & 0 & 0 & 1 & -1 & -1 \\
1 & 0 & -1 & 0 & 1 & 0 & 0 & 3 & -1 & -2 & 2 & 2 \\
-1 & 0 & 0 & 1 & 0 & -1 & 0 & -1 & 3 & 1 & -2 & -2 \\
-1 & 0 & 1 & 0 & -1 & 0 & 1 & -2 & 1 & 3 & -2 & -2 \\
1 & 1 & -1 & 0 & 1 & 1 & -1 & 2 & -2 & -2 & 4 & 3 \\
1 & 1 & -1 & 0 & 1 & 1 & -1 & 2 & -2 & -2 & 3 & 4
\end{array}\right)
$$

with automorphism group of order $2^{15} 3^{4}$ resp. $2^{21} 3$.

$L_{7}(2):\left(\begin{array}{cccccccccccccc}2 & 0 & 0 & 0 & 0 & 1 & 1 & 1 & 1 & 1 & 0 & 0 & -1 & 0 \\ 0 & 2 & 0 & 0 & 1 & 1 & 1 & 1 & 0 & 0 & 0 & 0 & 0 & 1 \\ 0 & 0 & 2 & 0 & 1 & 1 & 0 & 0 & -1 & 1 & 1 & -1 & 0 & 0 \\ 0 & 0 & 0 & 2 & -1 & 0 & -1 & -1 & 0 & 0 & -1 & -1 & 1 & 0 \\ 0 & 1 & 1 & -1 & 3 & 1 & 1 & 1 & 0 & 1 & 1 & 0 & 0 & 1 \\ 1 & 1 & 1 & 0 & 1 & 3 & 1 & 1 & 0 & 1 & 1 & 0 & -1 & 0 \\ 1 & 1 & 0 & -1 & 1 & 1 & 3 & 2 & 0 & 0 & 0 & 0 & -1 & 1 \\ 1 & 1 & 0 & -1 & 1 & 1 & 2 & 3 & 1 & 1 & 1 & 1 & -1 & 0 \\ 1 & 0 & -1 & 0 & 0 & 0 & 0 & 1 & 3 & 0 & 0 & 1 & 0 & 0 \\ 1 & 0 & 1 & 0 & 1 & 1 & 0 & 1 & 0 & 3 & 1 & 0 & 0 & 0 \\ 0 & 0 & 1 & -1 & 1 & 1 & 0 & 1 & 0 & 1 & 3 & 0 & -1 & -1 \\ 0 & 0 & -1 & -1 & 0 & 0 & 0 & 1 & 1 & 0 & 0 & 3 & -1 & -1 \\ -1 & 0 & 0 & 1 & 0 & -1 & -1 & -1 & 0 & 0 & -1 & -1 & 3 & 1 \\ 0 & 1 & 0 & 0 & 1 & 0 & 1 & 0 & 0 & 0 & -1 & -1 & 1 & 3\end{array}\right)$.

Automorphism group of order 2752512 .

$L_{8}(2): L_{8}(2)$ is the odd version of the Barnes-Wall lattice $B W_{16}$ (see [6]). It is unique by $[9$, Theorem 8$]$. 
$L_{1}(6):\left(\begin{array}{cccc}2 & -1 & -1 & 0 \\ -1 & 3 & 0 & -1 \\ -1 & 0 & 3 & -1 \\ 0 & -1 & -1 & 4\end{array}\right)$.

Automorphism group $C_{2}^{4}$.

$L_{2}(6):\left(\begin{array}{cccccccc}3 & 1 & 0 & 0 & 0 & 1 & 0 & -1 \\ 1 & 3 & 0 & 0 & -1 & 0 & -1 & 0 \\ 0 & 0 & 3 & 1 & 0 & -1 & 0 & -1 \\ 0 & 0 & 1 & 3 & 1 & 0 & -1 & 0 \\ 0 & -1 & 0 & 1 & 3 & 1 & 0 & 0 \\ 1 & 0 & -1 & 0 & 1 & 3 & 0 & 0 \\ 0 & -1 & 0 & -1 & 0 & 0 & 3 & -1 \\ -1 & 0 & -1 & 0 & 0 & 0 & -1 & 3\end{array}\right)$.

Automorphism group $S L_{2}(3) .2^{2}$ of order 96 .

$L_{1}(14)$ : Gram matrix $\left(\begin{array}{ll}3 & 1 \\ 1 & 5\end{array}\right) \perp\left(\begin{array}{ll}3 & 1 \\ 1 & 5\end{array}\right)$.

Automorphism group $D_{8}$.

\section{References}

[1] N.D. ElKIES, A characterization of the $\mathbb{Z}^{n}$ lattice. Math. Res. Lett. 2 no. 3 (1995), 321-326.

[2] N.D. Elkies, Lattices and codes with long shadows. Math. Res. Lett. 2 no. 5 (1995), 643651.

[3] M. Gaulter, Lattices without short characteristic vectors. Math. Res. Lett. 5 no. 3 (1998), $353-362$.

[4] C. L. Mallows, A. M. Odlysko, N. J. A. Sloane, Upper bounds for modular forms, lattices and codes. J. Alg. 36 (1975), 68-76.

[5] T. MiYake, Modular Forms. Springer (1989).

[6] G. Nebe, N.J.A. SloAne, A database of lattices. http://www.research.att.com/ njas/lattices

[7] H.-G. QuebBemann, Modular lattices in euclidean spaces. J. Number Th. 54 (1995), 190202.

[8] H.-G. QuebBemann, Atkin-Lehner eigenforms and strongly modular lattices. L'Ens. Math. 43 (1997), 55-65.

[9] E.M. Rains, N.J.A. Sloane, The shadow theory of modular and unimodular lattices. J. Number Th. 73 (1998), 359-389.

Gabriele NEBE

Abteilung Reine Mathematik

Universität Ulm

89069 Ulm, Germany

E-mail : nebe@mathematik.uni-ulm.de 